\title{
Memorability of Pre-designed \& User-defined Gesture Sets
}

\author{
Miguel A. Nacenta, Yemliha Kamber, Yizhou Qiang and Per Ola Kristensson \\ School of Computer Science, University of St Andrews \\ North Haugh, St Andrews, KY16 9SX, United Kingdom \\ \{mans, yk9,yq7, pok\}@st-andrews.ac.uk
}

\begin{abstract}
We studied the memorability of free-form gesture sets for invoking actions. We compared three types of gesture sets: user-defined gesture sets, gesture sets designed by the authors, and random gesture sets in three studies with 33 participants in total. We found that user-defined gestures are easier to remember, both immediately after creation and on the next day (up to a $24 \%$ difference in recall rate compared to pre-designed gestures). We also discovered that the differences between gesture sets are mostly due to association errors (rather than gesture form errors), that participants prefer user-defined sets, and that they think user-defined gestures take less time to learn. Finally, we contribute a qualitative analysis of the tradeoffs involved in gesture type selection and share our data and a video corpus of 66 gestures for replicability and further analysis.
\end{abstract}

\section{Author Keywords}

Gesture sets; gesture memorability; user-defined gestures.

\section{ACM Classification Keywords}

H.5.2.[Information interfaces and presentation]: User Interfaces-Input devices and strategies.

\section{INTRODUCTION}

Advances in user interface research have enabled the design of gesture sets that allow people to issue a wide range of commands by performing gestures that are recognized by the computer $[28,34,1,21,13]$. Some examples of gestures sets include the pen-based Rubine gesture set [28], Graffiti, Unistrokes [10] and the \$1 gesture set [36]. Recently, gesture sets have also been intensively studied for surfaces and other multi-touch devices $[8,35,14,16,25]$ and for 3D fullbody-motion tracking sensors [17,30,32,33].

There are many plausible advantages with gesture interfaces. For example, gestures do not take screen real estate or visually clutter the interface, they might be easier to associate with the intended actions, they can be invoked from multiple locations, and they are not orientation-dependent. The latter is useful in large-display collaborative scenarios [24]. Further, the industry interest in gestures interfaces is highlighted by the increasingly rich set of gestures available in touchpads and gestural interface platforms (e.g., [18]).

(c) ACM 2013. This is the author's version of the work. It is posted here for your personal use. Not for redistribution. The definitive Version of Record was published in the Proceedings of the SIGCHI Conference on Human Factors in Computing Systems (CHI '13), http://dx.doi.org/10.1145/2470654.2466142

The copy of record of the paper can be found in: http://dl.acm.org/citation.cfm?doid=2470654.2466142
One of the main factors that could determine the success of gesture sets in modern interfaces is whether the gestures can be effectively learned and remembered. Researchers investigating gestural interfaces have previously highlighted the importance of gesture memorability (e.g., [20]), but there is little empirical evidence regarding the memorability of gestures in general, and no evidence on the memorability of user-defined vs. pre-designed gestures, one of the toplevel design decisions that can affect memorability.

In this paper we empirically and analytically contribute to our understanding of the interrelationship and trade-offs between user-defined and pre-designed gestures. A key finding is that personalized gesture sets designed by the users themselves are significantly more memorable than pre-designed gestures that were, in our case, defined by two designers. This result was replicated in a series of three experiments that tested participants' recall of gesture sets for three feature-rich applications: an image editor, a web browser, and a word processor. The advantage of userdefined gesture sets is not due to the time required for users to create user-defined gestures, as one might initially expect. When controlling for time across the conditions, userdefined gestures were still significantly easier to remember.

In addition, participants significantly preferred user-defined gesture sets, and they thought creating user-defined gesture sets took less time than learning pre-designed gesture sets (when in fact the actual time difference between the two conditions was negligible). In general, users experienced user-defined gestures as easier, more fun and less effortful.

These results increase our understanding of gesture interfaces and show that it is well worth enabling users to design gestures for certain application-specific actions themselves. However, there are also situations when pre-designed gestures, designed either by a team of designers or elicited by users, are more suitable. We therefore provide an analysis of the trade-offs between user-defined and pre-designed gestures. In summary, our contributions are four-fold:

- We provide new empirical evidence on the memorability of gestures based on more than 100 hours of data gathered from 33 participants in three experiments.

- We present a qualitative analysis comparing user-defined, pre-designed, and stock gesture sets.

- We derive three guidelines for interface designers based on our empirical evidence.

- We share the data from the user studies to enable further analysis and to ensure replicability. 


\section{RELATED WORK}

Gestures are used for a variety of tasks, including writing text (e.g. [10,1,34,26]), issuing commands (e.g. [15,3,2, 5]), and modifying objects (e.g. [27,7]). See the recent survey by Zhai et al. [39] for a comprehensive review of 2D gesture interfaces. In order for gestures to be used they have to be designed. For this purpose, Long et al. [19] created $g d t$, the gesture design tool. It enables designers to create $2 \mathrm{D}$ gestures. Several tools for helping designers create gestures sets have been created thereafter, most recently Gesture Coder [21], \$N-protractor [1] and Proton [13].

However, for users to leverage gestures in the first place, they have to discover and learn how to apply them effectively. An early study investigated the immediate usability of Graffiti gestures and found that users can learn the entire gesture set within five minutes [24]. Later Wobbrock et al. [34] nuanced the concept of immediate usability by studying what they call the guessability of individual gestures. They proposed a procedure for designing and evaluating a highly guessable gesture set from participant data. It has later been pointed out that most tabletop gesture sets are pre-designed and that such gestures may not accurately reflect users' expectations [35]. Consequently, Wobbrock et al. [35] proposed a methodology for eliciting tabletop gesture sets from users and used this methodology to create a set of user-elicited gestures. A follow-up study showed that users preferred user-elicited gestures, and to a lesser extent, gestures proposed by 2-3 designers [25]. Gestures created by a single designer were the least preferred. Several others have later adopted the user-elicited paradigm to, for example, design gesture sets for mobile phones [14,30], interactive television $[33,12]$, multi-display environments [16] and smart-home environments [17]. A recently conducted study on user-elicited gesture sets found that users tend to generate gestures with familiar characteristics even when they are told to generate distinct and novel gestures [29].

Several systems have been developed to help users discover, learn and articulate gestures. Command Strokes [15] enables users to gesture textual representations of commands (e.g., the string "copy") on touchscreen keyboards. To aid gesture discoverability, it continuously recognizes gestures as they are articulated on the keyboard and visually presents the most likely commands to the user. Octopocus [3] is a system similar in spirit that helps users discover and articulate free-form gesture commands on touchscreens. In multi-touch and surface environments the ShadowGuides [8] system helps users learn multi-touch and whole hand gestures. For 3D gesture interfaces, LightGuide [32] projects gesture guidance hints directly onto the user's hand.

A relatively underexplored issue in gesture interfaces is memorability. In the context of text entry, Zhai and Kristensson [1] investigated how many unfamiliar gestures users can learn via a training program. Cockburn et al. [6] investigated whether inducing effort can improve gesture recall of single-stroke gestures. Inducing effort improved memorability but users found the effort-inducing gesture training interface less enjoyable and more frustrating. Later, in a study of strategies for teaching users gestures for surfaces, Freeman et al. [8] found that users remembered more surface gestures when using their ShadowGuides system compared to video-based assistance. Another memorability study was conducted by Appert and Zhai [2]. When testing users' ability to issue 14 commands, they found that they were able to recall more gestures than keyboard shortcuts.

Recently, as part of a series of four experiments, Kühnel et al. [17] investigated how well ten users could rate and learn seven (for them unfamiliar) user-elicited 3D gestures in a smart home environment. The gestures were performed by holding a mobile phone in one of their hands. While Kühnel et al. [17] do not report how many gestures users remembered, they conclude that gestures representing frequent non-complex actions tended to result in similarly elicited gestures, which in turn tended to be easier for the users to remember. Finally, Jansen [12] investigated three ways of teaching users a set of ten (for them unfamiliar) userelicited gestures for interactive television. Jansen [12] reports that users could correctly recall $61-71 \%$ of the gestures on average, depending on the teaching method.

\section{PRE-DESIGNED VS. USER-DEFINED GESTURE SETS}

For this work we distinguish between three main different classes of gesture sets: pre-designed, stock, and userdefined. We define each type and discuss their customizability, discriminability, consistency, transferability and awareness, and user time and effort.

Pre-designed. These are gesture sets that are created by designers for a particular application. Designers can take advantage of their expertise to create gestures that will be suitable for people (e.g., memorable, easy to perform), use their knowledge of the system's recognizer technology to improve recognition rates, and put effort towards creating strong mappings between gestures and actions [35,25]. We consider user-elicited gestures [35] as a special case of predesigned gesture sets. The gestures that comprise these sets are generated by a representative group of users in a study, generally at system design time, and are then carefully compiled and selected to form a consistent "agreed upon" gesture set that is still recognizable by the system (e.g., it does not assign identical gestures to several actions) [35]. We acknowledge that user-elicited gesture sets can differ from pre-designed gesture sets, and we discuss these differences in this section and in the discussion. However, the creation of such sets introduces significant complexity and requires a full additional phase of testing. Moreover, userelicited gesture set creation is still an evolving technique, with many variants [9]. We therefore decided not to include user-elicited gestures as a condition in our study, and leave this more fine-grained comparison for future research.

Stock. Systems can come pre-loaded with a generic stock gesture set that can be used by many applications, without 
specifically designed relationships between gestures and application actions. These sets might be useful for systems that have not been designed with a specific user interface in mind (e.g., legacy applications). An example of this approach is the MS Windows gesture API [23].

User-defined. Some systems enable individuals to define their own gestures for actions (e.g., [19]).

\section{Customizability}

The ability to create gestures that adapt to the context and needs of the individual can be an advantage of user-defined gesture sets. Customizability can impact accessibility (e.g., people with reduced right-hand mobility could create gestures that do not involve that hand), and enables adaptation to the individual's needs (e.g., frequent tasks are assigned to faster gestures). Customized gestures might also help leverage people's personal background (e.g., culture, personality, and experiences) to provide easier to remember personal associations. In contrast, pre-defined and stock gesture sets need to be learned. However, we do not have any evidence on whether user-defined or learned gestures are easier to remember. Our work addresses this question.

\section{Recognizer Discriminability}

Although gesture recognizer technology is constantly improving, recognizers are not perfect. Two gestures from a user-defined gesture set can seem very different to the user defining them, but may appear similar to the recognizer. As a result, recognizers for user-defined gesture sets need to be more sophisticated and may suffer from lower accuracies than recognizers for professionally-designed sets. Gesture designers can apply their expertise and invest more time in balancing human aspects and machine-discriminability.

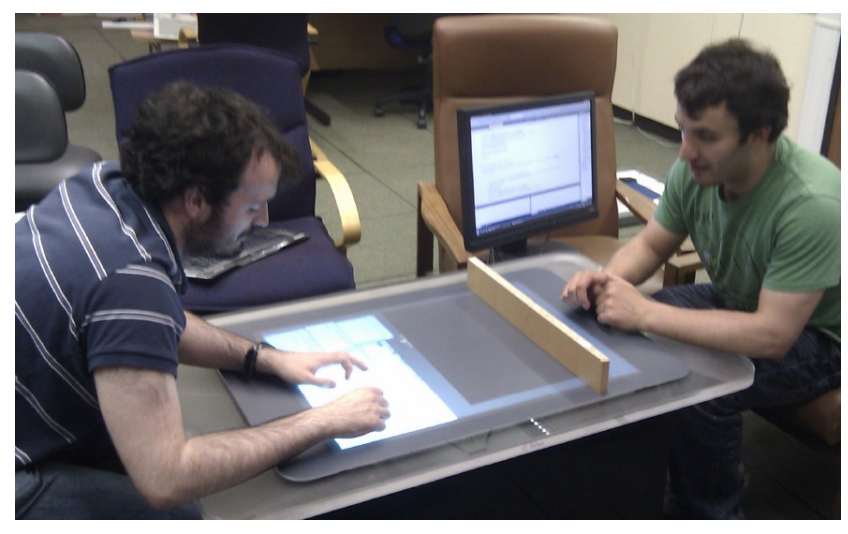

Figure 1. Experimental setup. The participant sat on the left and the experimenter sat on the right.

\section{Consistency}

Gesture sets designed by professionals are likely more consistent than those spontaneously generated by users. Consistency is a desirable property of interfaces in general, and it can be of increasing importance as the number of gestures increases. Consistently designed gesture sets can even form a grammar that enables the invocation of many actions through combinations of relatively few subgestures [4,37].

\section{Collaborative Awareness and Transferability}

Gestures are often necessary in multi-user scenarios; a critical aspect in $\mathrm{CSCW}$ is that collaborators can interpret and follow what others are doing (workspace awareness [11]). User-defined gestures will likely be harder to interpret by collaborators, whereas if everybody shares the same set they can follow other's activities more easily. A unified set of gestures that is common across applications also enables people to use the same gestures in multiple systems without having to personalize the system for a particular person.

\section{Effort/Time}

Learning a set of gestures requires a certain amount of time and effort, but coming up with gestures does take time and effort as well. More importantly, the user-perceived time and effort that has to be invested into learning or creating gesture sets might be crucial for the success of the interface. As part of our study we investigate the effort and time of learning pre-defined gestures vs. inventing new ones.

\section{EMPIRICAL EVALUATION}

The related work and analysis sections above highlight important unresolved questions about gesture memorability. Most importantly, can users remember gestures that they define themselves better than pre-designed or arbitrarily assigned sets? We designed a series of three studies to answer this question.

\section{EXPERIMENT 1}

\section{Apparatus}

The experiment was carried out on a Microsoft Surface 1.0 device running custom experimental software. The participant and the experimenter sat on opposite short ends of the surface (see Figure 1). The visible interface on the table was divided into two main areas (see Figure 2): the participant area (B- $85 \%$ of the display area), and the experimenter area $(\mathrm{A}-15 \%$ of the display area). The experimenter's area was occluded from the participant's line of sight by a small wooden vertical screen (see Figure 1).

The participant's area was divided into three sections. The participant video area (Figure 2.E) was used to present gesture videos to the participant, the action area (2.G), where the participant would reproduce, create, or remember gestures, and the action presentation area (2.F), which displayed the initial state, name of the action, and intended result of the gesture. The experimenter's area contained an experimenter reference video widget (2.C) and buttons to control the flow of the experiment and to log if the participant's gesture was correct, almost correct, or incorrect (2.D). The Surface was touch-enabled, but the gestures performed by the participant did not have to contain touches on the surface (i.e., they could be free-form above the table), and therefore no automatic sensing of gestures was provid- 
ed. The correctness of the gestures was determined by the experimenter by comparing the participant's gesture with a pre-existing video that was not visible to the participant. A camera overlooking the action area from behind the participant's left shoulder recorded the user-defined gestures.

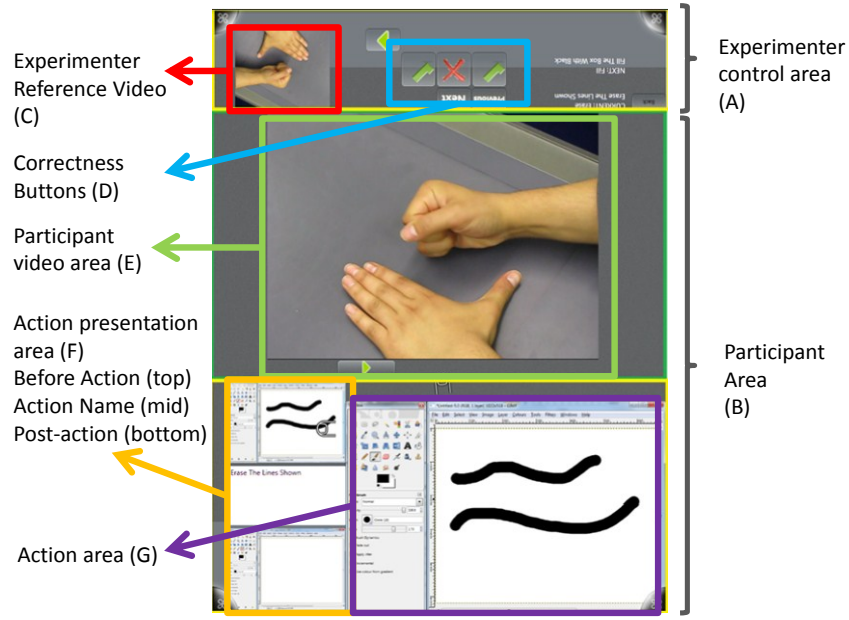

Figure 2. Annotated snapshot of the experimental interface.

The action being shown in areas $F$ and $G$ is "delete lines".

\section{Applications, Action Sets and Gesture Sets}

The experiment followed a within-subjects design in which participants learned and remembered gestures in three order-balanced conditions: user-defined, pre-defined, and random gestures. For experimental purposes, three sets of 16 actions were selected for each of three applications, simulating activities that could take place on or over a multitouch surface: image editing, web browsing and word processing. These three applications were selected because they contain many possible actions (e.g., make selected text bold, access bookmark menu, create a new layer), and are familiar to most computer users. We needed familiar applications that were distinct from each other in order to prevent the participant confounding the results by not being able to remember what an action meant. We believe that the set of actions that we selected are representative of most existing or future software-supported activities. We avoided similarity within and across applications when selecting actions. For example, because we selected the "go to previous page" action in the web browser, we chose to avoid testing an "undo" action for the image editor and the word processor. The full list of actions is in the video figure.

Each participant carried out three experimental cycles of learning and gesture memory testing, one in each condition (user-defined, pre-designed, and random), and with a different application (image editor, web browser, and word processor). The applications were always visited in the same order, but condition balancing forced applicationcondition combinations to appear an equal number of times.

\section{Pre-designed Gesture Set Condition}

The pre-designed condition corresponds to a gesture set designed by two of the authors of the study. For each action in a group of 16, a free-form gesture was designed that complied with the following conditions:

- The gesture was specific enough to be distinguishable from simple touch manipulations such as pinching;

- The gesture was distinguishable from other gestures within and across gesture sets;

- The gesture could contain any sequence of free-form movements in the air, or hand contacts with the surface.

In addition to these conditions, the designers applied their experience in the design of multi-touch gesture sets (e.g., [31]) to achieve reasonable gestures that were consistent with the task in three iterations.

\section{User-defined Gesture Set Condition}

In the user-defined gesture set condition, participants created their own gestures. Participants were given an image of the initial state, the name of the action, and the final state, and they had unlimited time to create a gesture for the action. Once they had decided on a gesture, they would reproduce it once to be video-recorded by the experimenter. The experimenter reminded the participants multiple times that gestures did not have to be limited to contact with the surface (e.g., they could be articulated in the air).

\section{Random Gesture Set Condition}

The random gesture set condition was included to serve as a baseline comparison. It also represents stock gesture sets. Actions were assigned to gestures from the pre-designed set of gestures that participants did not get to see that corresponded to a different application. For example, if the participant went through the pre-defined, user-defined and random experiment order, they would have to learn the predefined gestures for the browser first, then design their own gestures for the word processor actions, and finally learn a randomly assigned set of gestures that were originally designed for the word processor, only randomly assigned to image editor actions. This approach guarantees that the gestures were plausible, not seen by the participant before or after, and not specifically created for the application.

\section{Procedure}

Six male volunteers (age 22 to 28 ) participated in the study. After providing consent and before starting the first phase of the first condition, participants went through a short demo in which they experienced a short version of the basic procedure with a set of actions not included in the rest of the experiment ("cut", "copy", "paste", "print"). Participants came four days to the lab to perform a LearnReinforce-Test cycle for each of the conditions. Testing for a learned set would always happen on the next day. For example, if L,R,T represent the three phases of each condition detailed below, a participant in the user-defined (UD), pre-designed (PD), random (RD) order would do the following sequence: Day $1 \mathrm{~L}_{\mathrm{UD}}+\mathrm{R}_{\mathrm{UD}}$; Day $2 \mathrm{~T}_{\mathrm{UD}}+\mathrm{L}_{\mathrm{PD}}+\mathrm{R}_{\mathrm{PD}}$; Day $3 \mathrm{~T}_{\mathrm{PD}}+\mathrm{L}_{\mathrm{RD}}+\mathrm{R}_{\mathrm{RD}}$; Day $4 \mathrm{~T}_{\mathrm{RD}}$. Days 1 and 4 took about 40 minutes, 2 and 3 took approximately 1 hour. 


\section{Learning/Creation}

During this phase, participants were taught a gesture set. For each gesture in a series of 16, the interface would present an action in the action presentation area (Figure 2.F), which consists of an initial state, an action name, and a final state. For example, the "make bold" action would show a regular line of text in the top of the action presentation area (top of part F in Figure 2), the words "make bold" in the middle, and the same text but in a bold typeface in the bottom. Then, a video would play in the participant video ar$e a$, showing the specific physical gesture for the current action. The video could be played repeatedly if the participant was not sure about what gesture was shown. Finally, the participant would reproduce the gesture in the action area; if the experimenter recognized the gesture as correct, this would trigger the same kind of result in the action area as was shown in the action presentation area (e.g., turning the existing text to bold).

The transitions between these stages were triggered by the participant, who had to touch on the different areas of the screen to progress through all the phases except the last, where the experimenter made sure that the reproduced gesture corresponded to that shown on the video. If not correctly reproduced, the experimenter would play the video again and ask the participant to repeat.

In the user-defined condition, participants did not have to learn a gesture set, but instead create one. The gesture creation procedure was similar to the learning process, but instead of watching a pre-recorded video, participants were given unlimited time to design a gesture which, when a stable gesture was achieved, was video-recorded.

\section{Reinforcement}

Immediately after the full set of gestures was learned or created, the same set of gestures was tested. This phase was introduced to simulate real-world learning circumstances more realistically, where learning a gesture would not take place in isolation, but instead would happen within the context of actual use and application of the learned gesture set.

The reinforcement process was similar to the learning phase, but participants would try to recall and reproduce the gesture corresponding to an action first, after which they would be notified of its correctness, and then shown the video of the correct gesture. In this phase, the gestures were presented in the same order as during the learning phase, and the correctness of the answer was judged by the experimenter and the result was logged by the system.

\section{Next-day Testing}

We collected the main memorability measure of the study in a session that took place the day after the corresponding learning phase. This testing phase was identical to the reinforcement phase, except that no video reminding participants of the right gesture was presented at the end of the trial, and the participants were not informed of the correct- ness of their gesture (to avoid possible cross-gesture recall effects). The correctness of the gesture was judged by the experimenter with the help of the video used to teach the gesture, which was visible only to him. If the experimenter had any trouble seeing the participant's execution of the gesture, he asked the participant to repeat the gesture again.

\section{Measurements and Analysis}

The main measure of the study was the number of correct recalls of gestures during the test phase. A gesture would be judged as correctly recalled if it involved the same parts of the hands, the same number of hands, fingers and contacts, and the same overall movement shapes and timing. If the reproduction of the gesture was similar, but with some noticeable difference (e.g., a different number of fingers, different fingers used, or different number of taps) the gesture was considered a close gesture. The distinction between regular recall errors and close errors helps us distinguish between two types of memorability problems in gestures: association errors (failure to associate the action with the correct gesture), and partial gesture errors (failure to recall the specifics of the gesture). The same criteria were applied to the measures during the reinforcement phase. Additionally, we measured the time to respond in both the reinforcement and test phases.

Since the gesture learning and design process was mostly participant-driven and the time spent defining or learning gestures was variable, we also measured the time that each participant spent learning or defining each gesture.

Visual inspection of the recall distributions revealed that these were plausibly normal. All time-based tests were performed on log-transformed data. No sphericity tests discounted sphericity. All post-hoc tests were corrected for multiple comparisons using Holm-Bonferroni corrections.

\section{Results}

\section{Next-day tests}

The average recall rates on the next day were $97 \%$ for the user-defined gesture set, $82 \%$ for pre-designed, and $52 \%$ for random (see Figure 3). A repeated-measures ANOVA of the recall rates showed a significant effect of the conditions $\left(\mathrm{F}_{2,10}=58.7, \mathrm{p}<0.001, \eta_{\mathrm{p}}{ }^{2}=0.92\right)$, which was also reflected in significant pairwise comparisons (all $\mathrm{p}<0.013$ ).

To analyze the source of errors we performed a two-way RM-ANOVA of the number of errors with type of error (association, partial gesture) and gesture set (user-defined, pre-designed, random) as factors. The analysis showed significant main effects of both factors $\left(\mathrm{F}_{2,10}=58.7, \mathrm{p}<0.001\right.$, $\left.\eta_{\mathrm{p}}{ }^{2}=0.92 ; \mathrm{F}_{1,5}=9.3, \mathrm{p}=0.028, \eta_{\mathrm{p}}{ }^{2}=0.65\right)$ as well as an interaction effect $\left(\mathrm{F}_{2,10}=10.8, \mathrm{p}=0.003, \eta_{\mathrm{p}}{ }^{2}=0.68\right)$. The interaction indicates that the pattern of association errors might be different to that of partial gesture errors; to further investigate that, we ran separate ANOVAs for each type of error, which were both significant $\left(\mathrm{F}_{2,10}=7.0, \mathrm{p}=0.013\right.$, $\left.\eta_{\mathrm{p}}{ }^{2}=0.58 ; \mathrm{F}_{2,10}=73.1, \mathrm{p}<0.001, \eta_{\mathrm{p}}{ }^{2}=0.94\right)$. However, the 

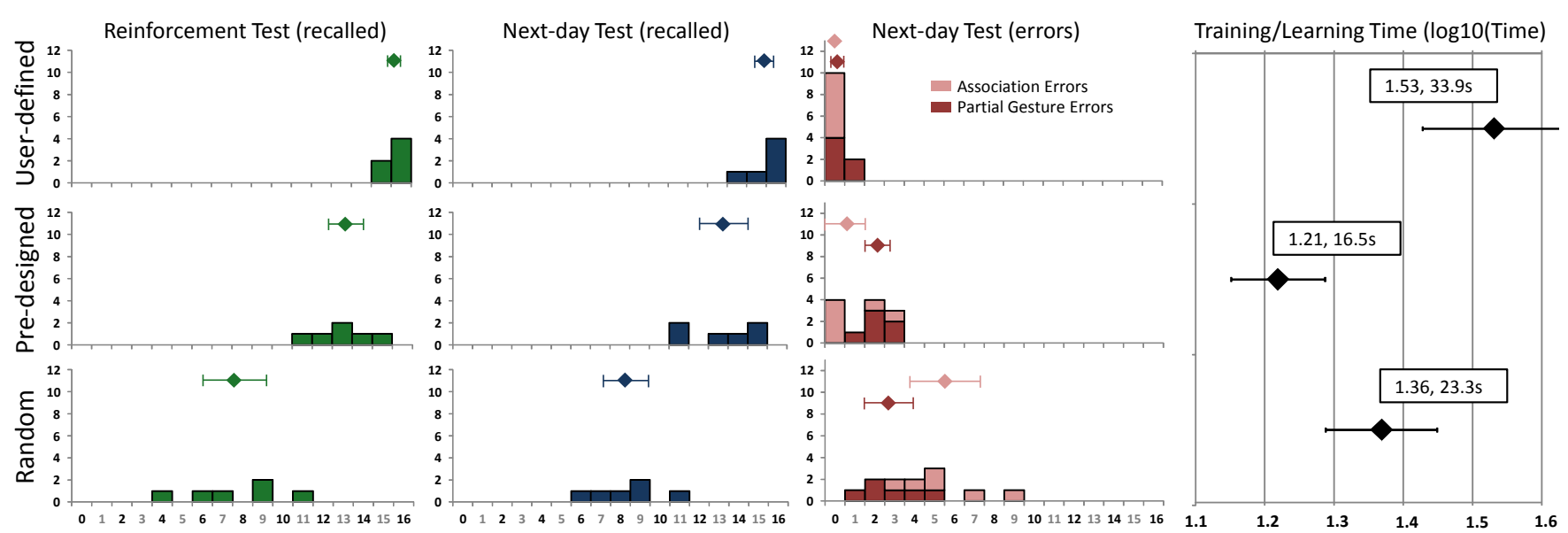

Figure 3. Results of Experiment 1. By column, (1) histogram, \# of participants correctly recalling $\mathrm{x}$ gestures (x axis) during the reinforcement test, (2) next-day correct recalls, (3) histogram of errors (by association/partial gesture), (4) Learning/training times (Log10). Diamonds are averages. Error bars show 95\% confidence intervals.

pattern in the post-hoc pairwise comparisons was different for each error type. For partial gesture errors only the differences between the random gesture set and the predesigned and user-defined sets approached significance after adjusting for multiple comparisons $(\mathrm{p}=0.017 ; \mathrm{p}=$ 0.027), whereas for association errors all pairwise comparisons were significant (all $p<0.05$ ), with user-defined gestures having the least amount of average association errors (0.5), followed by pre-designed (1.3) and random (5.5).

\section{Reinforcement tests and learning/training time}

The proportion of correct answers in the reinforcement phase follows the same pattern as for the next-day test: $98 \%$ correct for user-defined, $81 \%$ for pre-designed and $47 \%$ for random. In a parallel analysis, an RM-ANOVA of the recall rates showed a statistically significant difference between conditions $\left(\mathrm{F}_{2,10}=38, \mathrm{p}<0.001, \eta_{\mathrm{p}}{ }^{2}=0.88\right)$, which was also reflected in significant pairwise tests (all $\mathrm{p}<0.008)$.

Finally, we compared the amount of time that participants spent creating gestures in the user-defined condition vs. learning the gestures in the other two. An RM-ANOVA of the log-transformed times showed differences in training time $\left(\mathrm{F}_{2,10}=17.9, \mathrm{p}<0.001, \eta_{\mathrm{p}}{ }^{2}=0.78\right)$. It took participants $42.1 \mathrm{~s}$ on average to define a gesture for the user-defined gesture set, whereas it took $25.9 \mathrm{~s}$ and $17.9 \mathrm{~s}$ to learn gestures from the random and pre-defined sets respectively (see Figure 3, column 4 for the log-transformed averages).

\section{Summary and Discussion of Experiment 1}

The results of this experiment show a potentially large advantage in memorability of the user-defined gesture set ( $15 \%$ better recall than pre-designed on the next day, $17 \%$ right after the test), with a very clear disadvantage of the random set. Additionally, the error analysis indicates that association errors vary much more across types of gesture sets than partial gesture errors.

However, the time analysis showed that it takes approximately twice the time to create a gesture than to present it to the participant, at least in our experiment. This may have introduced a confound: it is possible that the advantages in memorability of the user-defined gesture set were due to the longer times participants spent creating it. Additionally, the data displayed in Figure 3 also shows a possible ceiling effect in the measures for the two most memorable gesture sets. Finally, the low number of participants is also a concern. Even though the significant results are unlikely to have been due to chance, the small sample size might have affected the reliability of the results.

\section{EXPERIMENT 2}

We designed a second experiment to address the above issues which a) included more participants, b) established a better control of the learning/creation time by forcing two consecutive repetitions of each gesture's learning phase (pre-designed and random), and c) reduced ceiling effects by including larger gesture sets. Additionally, to avoid possible effects due to sequential memorization, the tests used different orders for the training sequences and the test sequences. Below we detail the changes in gesture sets and procedure with respect to the first experiment; all other elements of the study where kept constant.

\section{Applications, Action Sets and Gesture Sets}

The three applications were the same as in the first experiment, as were the three conditions. The action and gesture sets of the first experiment where expanded with six new gestures per application to reach a total of 22 per condition. The videos of the 66 gestures are in the video figure.

\section{Procedure}

Nine participants ( 6 males and 3 females; age 20 to 40 ) participated in the study for compensation. The procedure was identical to that described for the previous experiment, except for: 1) the phases took longer due to the larger number of gestures, 2) the reinforcement and testing used a randomized order of gestures, 3) the learning of each gesture for the random and pre-designed conditions was repeated 
twice to enable comparable times spent learning the predefined and random gestures and creating the user-defined gestures, and 4) participants were incentivized to remember as many gestures as possible through a top-performer bonus. No participants had participated in the first experiment.

\section{Results}

\section{Next-day tests}

The average recall rates on the next day were $94 \%$ for userdefined gestures, $89 \%$ for pre-designed gestures, and $55 \%$ for random gestures (see Figure 4). An RM-ANOVA of the recall rates showed a significant difference between gesture sets $\left(\mathrm{F}_{2,16}=44.4, \mathrm{p}<0.001, \eta_{\mathrm{p}}{ }^{2}=0.85\right)$. Post-hoc pairwise comparisons were significant between the random and both the user-defined and the pre-designed conditions $(\mathrm{p}<$ 0.001 ), but not between user-defined and pre-designed.

An analysis of the distribution of the errors through a twoway ANOVA, with gesture set and type of error as main factors revealed significant effects of the gesture set $\left(\mathrm{F}_{2,16}=\right.$ $\left.44.4, \mathrm{p}<0.001, \eta_{\mathrm{p}}{ }^{2}=0.85\right)$, type of error $\left(\mathrm{F}_{1,8}=16, \mathrm{p}<\right.$ $\left.0.004, \eta_{\mathrm{p}}{ }^{2}=0.67\right)$, as well as a significant interaction of the two $\left(\mathrm{F}_{2,16}=48.1, \mathrm{p}<0.001, \eta_{\mathrm{p}}{ }^{2}=0.85\right)$. A further analysis of the two error types in separate ANOVAs for each type of error showed that association errors were significantly different between conditions $\left(\mathrm{F}_{2,16}=50.9, \mathrm{p}<0.001, \eta_{\mathrm{p}}{ }^{2}=\right.$ $0.86)$, whereas partial gesture errors were not $\left(\mathrm{F}_{2,16}=1.6, \mathrm{p}\right.$ $\left.=0.24, \eta_{\mathrm{p}}{ }^{2}=0.16\right)$. This shows that most of the variability in correctly recalled gestures across gesture sets can be explained by different rates of association errors.

\section{Reinforcement tests and learning/training time}

Recall rates in the test immediately after the initial learning phase were $92 \%$ for user-defined, $71 \%$ for pre-designed, and $47 \%$ for the random condition (see Figure 4 ). The omnibus repeated-measures ANOVA showed significant differences between conditions $\left(\mathrm{F}_{2,16}=29.9, \mathrm{p}<0.001, \eta_{\mathrm{p}}{ }^{2}=\right.$ 0.79 ). The pairwise tests were all significant (all $\mathrm{p}<0.015)$.

As a control, an analysis of the time spent learning or creating gestures for the three gesture sets revealed no statistically significant differences $\left(\mathrm{F}_{2,16}=0.30, \mathrm{p}=0.74, \eta_{\mathrm{p}}{ }^{2}=0.04\right)$.

\section{Summary and Discussion of Experiment 2}

The second experiment confirmed the results of the first experiment, with the additional assurance that the time invested in learning and creating was successfully controlled for across conditions. However, an important exception is that the user-defined and pre-defined gesture set were not statistically different in the next-day tests. This might again be explained as a ceiling effect. Although we tried to avoid ceiling effects by expanding the number of gestures, we also increased the learning time of pre-defined and random sets to make learning time equivalent to gesture creation time in the user-defined condition. This might have increased recall rates for the learned gesture sets, reintroducing a ceiling effect that precluded a significant difference between the pre-designed and user-defined sets.

An alternative explanation would be that the order randomization of tests introduced in the second experiment affected each condition in a different way. Although this is unlikely, ruling out this possibility requires a third experiment.

\section{EXPERIMENT 3}

This study addresses the limitations of the second experiment, but we also added changes that would cast light on additional issues. We doubled the number of participants to increase the power of the statistical tests and to avoid possible Type II errors. Additionally, we were interested in how recall rates of the different types of gesture sets would be affected by a longer period between learning and testing, which generalizes the results for gestures that are not immediately reinforced. Finally, besides the actual recall rates and times to learn/create, it is important to know whether participants perceive differences in effort, learnability, and time between sets, so we added questionnaires to elicit participants' subjective experiences.

\section{Procedure}

18 participants ( 8 female; age 20 to 39 ) participated in the study for compensation. The procedure was identical to the second experiment except for the following elements: 1) participants only reinforced half of the gestures immediately after the learning session (11 out of 22 gestures, random-

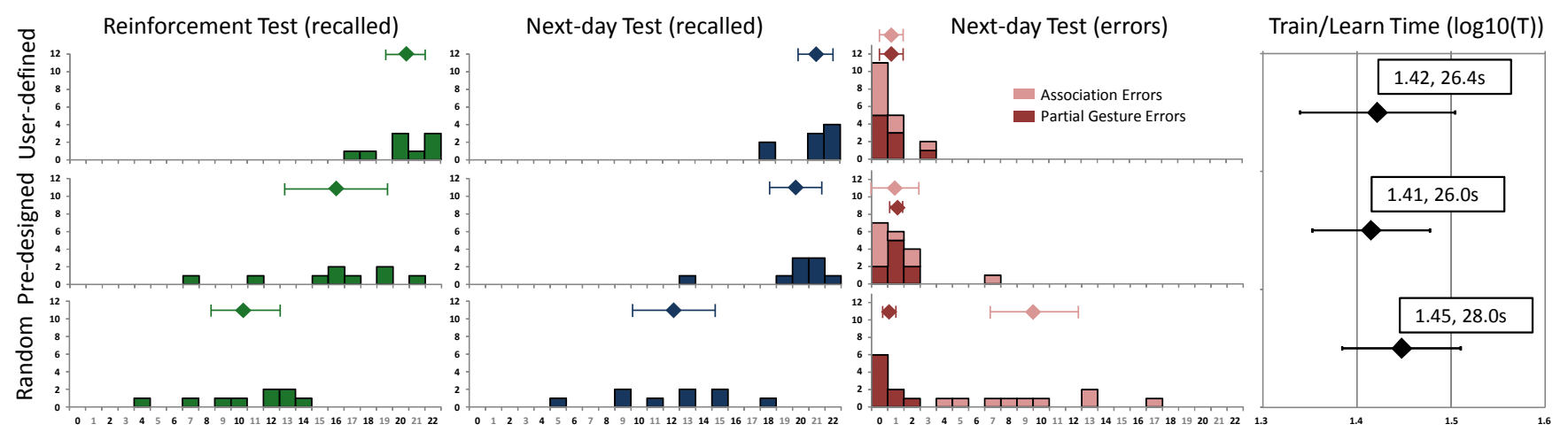

Figure 4. Results of Experiment 2. By column, (1) histogram, \# of participants correctly recalling $x$ gestures ( $x$ axis) during the reinforcement test, (2) next-day correct recalls, (3) histogram of errors (by association/partial gesture), (4) Learning/training times (Log10). Diamonds are averages. Error bars show $95 \%$ confidence intervals. 
ly selected), 2) participants filled out a questionnaire with four Likert-scale questions after each gesture set's next-day test session, 3) participants filled out a final questionnaire where they ranked the three gesture sets in terms of learning difficulty, recall difficulty, perceived time learning/creating the gestures, and fun. Participants were also incentivized. None of them had participated in any of the previous experiments. A different experimenter ran this study.

\section{Results}

\section{Next-day tests}

Average recall rates on the next day were $79 \%$ for userdefined gestures, 55\% for pre-designed and 25\% for random (see Figure 5). The reinforced (and non-reinforced) gestures were recalled in $85 \%(72 \%)$ of the cases for userdefined, $68 \%$ (41\%) for pre-designed, and 38\% (11\%) for the random set. Overall values might have been lower due to the change of experimenter between experiments $1 / 2$ and 3 , but the same criteria were applied for all correctness judgments within each experiment. Differences between conditions were confirmed by a two-way RM-ANOVA with gesture set and reinforcement as factors, showing significant main effects for both factors (gesture set: $\mathrm{F}_{2,34}=$ 109.2, $\mathrm{p}<0.001, \eta_{\mathrm{p}}{ }^{2}=0.87$; reinforcement: $\mathrm{F}_{1,17}=86.6, \mathrm{p}<$ $\left.0.001, \eta_{\mathrm{p}}{ }^{2}=0.84\right)$. Post-hoc pairwise comparisons were all significant for both factors (all $\mathrm{p}<0.001$ ).

Analyzing the distribution of the errors via a two-way ANOVA, with gesture set type and type of error as main factors) shows main effects of the gesture set $\left(\mathrm{F}_{2,34}=109.2\right.$, $\left.\mathrm{p}<0.001, \eta_{\mathrm{p}}{ }^{2}=0.86\right)$, type of error $\left(\mathrm{F}_{1,17}=59.3, \mathrm{p}<0.001\right.$, $\left.\eta_{\mathrm{p}}{ }^{2}=0.77\right)$, as well as an interaction of the two $\left(\mathrm{F}_{2,34}=50.7\right.$, $\left.\mathrm{p}<0.001, \eta_{\mathrm{p}}{ }^{2}=0.75\right)$. Due to the interaction, we further analyzed the two error types in separate ANOVAs for each type of error, which showed that association errors were significantly different between conditions $\left(\mathrm{F}_{2,34}=90.9, \mathrm{p}<\right.$ $\left.0.001, \eta_{\mathrm{p}}{ }^{2}=0.84\right)$, whereas partial gesture errors were not
$\left(\mathrm{F}_{2,34}=2.8, \mathrm{p}=0.074, \eta_{\mathrm{p}}{ }^{2}=0.14\right)$. This shows that most of the variability in correctly recalled gestures can be explained due to association errors.

\section{Reinforcement tests and learning/training time}

The average recall rates on the reinforcement test (same day) followed the same pattern as the next-day results, but, as expected, with lower proportions: $76 \%$ for user-defined, $56 \%$ for pre-designed and 33\% for random. A repeated measures ANOVA showed that the type of gesture set was significant $\left(\mathrm{F}_{2,34}=29.8, \mathrm{p}<0.001, \eta_{\mathrm{p}}{ }^{2}=0.63\right)$ as were all post-hoc comparisons between gesture sets (all $\mathrm{p}<0.005)$.

Our control of the time spent learning/creating gestures was successful; there were small differences in the logtransformed time averages between the three conditions (user-defined 26.9s, pre-designed 29.8s, and random $31.2 \mathrm{~s}$ - see Figure 5). The ANOVA was significant $\left(\mathrm{F}_{2,34}=\right.$ $\left.3.69, \mathrm{p}=0.035, \eta_{\mathrm{p}}{ }^{2}=0.18\right)$ but the post-hoc tests only showed a difference between user-defined and random $(\mathrm{p}=$ 0.031). The gesture set type that participants spent the most time learning was the one that had the worst recall.

\section{Subjective measures}

Participants perceived user-defined gestures as requiring less concentration to create, being easier to remember, and more fun. In the final questionnaire, participants ranked user-defined as easiest to learn, remember, less timeconsuming and most fun. The non-parametric omnibus comparisons (Friedman) and post-hoc tests (Wilcoxon signed-ranks) are reported in Table 1. Finally, in a question asking about overall preference 17 out of 18 participants chose user-defined as their favorite set.

\section{Summary and discussion of Experiment 3}

This experiment provided the statistical power necessary to strengthen the findings from Experiment 1, without the possible confound of learning time: user-defined gestures are

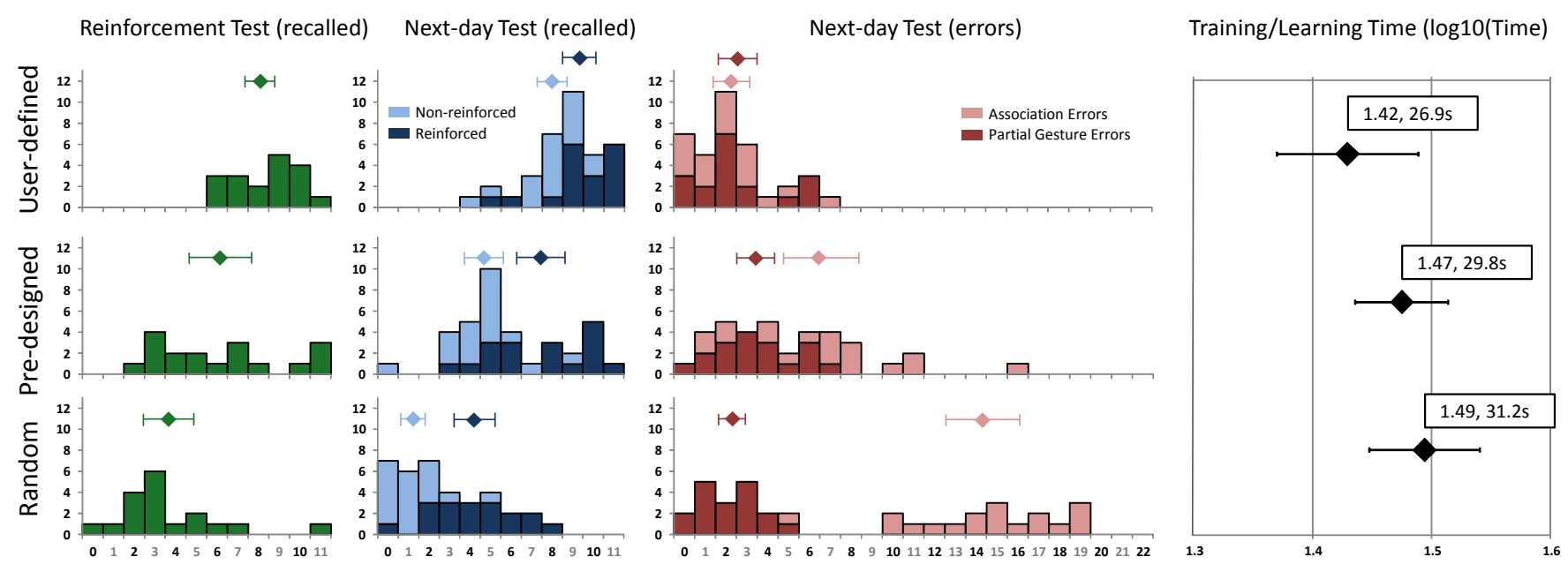

Figure 5. Results of Experiment 3. By column, (1) histogram, \# of participants correctly recalling $x$ gestures (x axis) during the reinforcement test, (2) next-day correct recalls (by reinforced/non-reinforced), (3) histogram of errors (by association/partial gesture), (4) Learning/training times (Log10). Diamonds are averages. Error bars show $95 \%$ confidence intervals. 
more memorable than pre-designed or random gestures, and random gestures are difficult to remember across the board.

\begin{tabular}{|c|c|c|c|c|c|c|c|c|}
\hline \multirow[b]{2}{*}{ Question } & \multicolumn{2}{|c|}{ Omnibus } & \multicolumn{3}{|c|}{ Medians } & \multicolumn{3}{|c|}{ Post-hoc Sig } \\
\hline & $\chi^{2}(2)$ & Sig. & UD & PD & $\mathrm{RD}$ & UD-PD & UD-RD & PD-RD \\
\hline Concentration to learn $\downarrow 1=$ low & 12.2 & 0.002 & 5 & 6 & 7 & 0.001 & 0.01 & 0.17 \\
\hline Easy to remember $1=$ very easy & 22.5 & $<0.001$ & 3 & 4.5 & 6.5 & 0.01 & $<0.001$ & 0.001 \\
\hline Ease of articulation $\downarrow 1$ = very easy & 16.2 & $<0.001$ & 3 & 3 & 4.5 & 0.8 & 0.001 & 0.01 \\
\hline Fun $\downarrow 1=$ very boring & 12.8 & 0.002 & 7 & 6 & 3.5 & 0.01 & 0.001 & 0.03 \\
\hline Difficulty learning (rank) 1 = hardest & 21 & $<0.001$ & 3 & 2 & 1 & 0.088 & $<0.001$ & 0.001 \\
\hline Diff. remembering (rank) $1=$ hardest & 18.1 & $<0.001$ & 3 & 2 & 1 & 0.16 & $<0.001$ & 0.001 \\
\hline Learning time (rank) $1=$ longest & 24.1 & $<0.001$ & 3 & 2 & 1 & 0.02 & $<0.001$ & 0.001 \\
\hline Fun (rank) $1=$ least fun & 18.8 & $<0.001$ & 3 & 2 & 1 & 0.01 & $<0.001$ & 0.01 \\
\hline
\end{tabular}

Table 1. Subjective test analyses. Medians are reported in green, yellow, red, for best, medium, worst set. Purple cells indicate statistically significant post-hoc comparisons.

The introduction of reinforced and non-reinforced gesture sets also shows that the differences between conditions hold for longer periods between learning and recall, which might be more common in real scenarios. As expected, reinforced gestures are more likely to be recalled in the next day than non-reinforced gestures.

The analysis of errors supports that the main differences in gesture set memorability can be attributed to differences in association errors, whereas the amount of partial gesture errors does not seem affected by the type of gesture set.

Subjective measures indicate that participants perceived user-defined gesture sets as easier to learn, more fun, and less effortful. They also thought that they took less time to create and preferred them overall.

\section{DISCUSSION}

The data from our experiments provides strong support for the use of user-defined gesture sets over pre-designed and random sets. Overall, we can expect recall rate differences between user-defined and pre-designed gestures of up to $24 \%$. The differences are also consistent across different time lapses. The increased memorability, in combination with other natural advantages of user-defined gestures (e.g., accessibility, optimization for the task) and the clear advantages perceived by users suggest that user-defined gestures can be a good choice in many situations. We conjecture user-defined gestures are more memorable because they allow individuals to more effectively take advantage of pre-established associations to their personal memories than what can be done by designers generically for all users.

Naturally, there are many scenarios where user-defined gestures might not be applicable or desirable; for example, when people's awareness of other's gestures is important for the task, when the gestures are common to a large set of applications (e.g., cut, paste), and if recognizers cannot reliably recognize user-defined gestures. However, the rate of improvement in sensing and recognizer technology suggests that the technical limitations will be less relevant in the near future. It will still be the designer's call whether to support pre-designed or user-defined gesture sets, but the designer might also want to create systems that can switch from one mode to another.

For designers of gestures, our data suggests that partial gesture errors are less important than association errors. The associative link between action and gesture seems to be the key factor to make a gesture set memorable, as it is also highlighted by the poor performance of random gestures. Designing gestures without knowing the actions they correspond to (stock gestures) seems generally unadvisable.

\section{Limitations and Future Work}

Our experiments compared the memorability of userdefined sets to gesture sets designed by two authors. We believe that the latter are representative of the gesture sets that other designers will create. An empirical evaluation of the representativeness of these sets is difficult practically and methodologically, and falls out of our scope. It is possible that a different designer could achieve a more memorable gesture set, but the large differences found in the study still show a significant advantage of user-defined sets.

Similarly, our experimental design did not compare userelicited sets. Whether user-elicitation (a sub-set of predesigned sets) can improve memorability deserves attention, but we do not anticipate user-elicited sets to have a significant advantage in this respect. Morris et al. [25], found that users preferred gestures that were created by more people, but the differences were relatively small (a 0.75 absolute difference on a 7-point Likert scale).

Our experiments are somewhat different from real-world gesture-learning contexts where gestures are learned while doing other tasks and are often used in sequences. Our research is a first step, but eventually our results will need to be confirmed in less controlled studies. Similarly, we chose applications for their familiarity. Applications other than browsers, image and word processors might be more common on surfaces in the future.

There are other important aspects of gesture sets that might also affect gesture memorability. For example, the type of gesture (e.g., deictic, literal), the input technology (surface, in-air), and cultural background require further study.

\section{CONCLUSIONS}

Gestures can be useful to invoke actions in interfaces with gesture recognizing capabilities. One of the most important aspects of gesture sets is memorability, since forgotten gestures can cause errors, increase frustration, and might prevent the adoption of gesture-based user interfaces. In this work we present a series of three studies investigating the memorability of three types of gesture sets: user-defined, pre-designed, and randomly assigned. The results show that user-defined gesture sets are more memorable than predesigned (up to $44 \%$ more gestures recalled), and they are considered less effortful, less time-consuming, and are preferred by people. In conjunction with our qualitative analysis, we can offer the following recommendations: 
- When not prevented by the context of use or the reliability of the recognizer, enable user-defined gesture sets.

- To design a gesture set for better memorability, consider closely its relationship with the action that it invokes, rather than the specific features of the gesture itself.

- Instead of using stock gesture sets, consider gestures designed for specific actions, or user-defined gestures.

\section{ACKNOWLEDGEMENTS}

This work was supported by the Engineering and Physical Sciences Research Council (grant number EP/H027408/1) and the Scottish Informatics and Computer Science Alliance. Yizhou Qiang's internship was supported by the School of Computer Science at the University of St Andrews. We thank Uta Hinrichs and Katherine Skipsey for their assistance.

\section{REFERENCES}

1. Anthony, L., Wobbrock, J.O. \$N-protractor: a fast and accurate multistroke recognizer. In Proc GI '12, 117-120.

2. Appert, C., Zhai, S. Using strokes as command shortcuts: cognitive benefits and toolkit support. In Proc. CHI '09, 22892298.

3. Bau, O., Mackay, W.E. OctoPocus: A dynamic guide for learning gesture-based command sets. In Proc. UIST '08, $37-$ 46.

4. Baudel, T., Beaudouin-Lafon, M. 1993. Charade: remote control of objects using free-hand gestures. Commun. ACM 36, 7, 28-35.

5. Bragdon, A., Zeleznik, R., Williamson, B., Miller, T. and LaViola, J., Joseph J. GestureBar: Improving the approachability of gesture-based interfaces. In Proc. CHI '09, 22692278.

6. Cockburn, A., Kristensson, P.O., Alexander, J, Zhai, S. Hard lessons: effort-inducing interfaces benefit spatial learning. In Proc CHI' 07 , 1571-1580.

7. Cohé, A. Hachet, M. Understanding user gestures for manipulating 3D objects from touchscreen inputs. In Proc. GI '12, 157-164.

8. Freeman, D., Benko, H., Morris, M.R., Wigdor, D. ShadowGuides: visualizations for in-situ learning of multi-touch and whole-hand gestures. In Proc. ITS '09, 165-172.

9. Frisch, M., Heydekorn, J., Dachselt, R. Investigating multitouch and pen gestures for diagram editing on interactive surfaces. In Proc. ITS '09, 149-156.

10. Goldberg, D. and Richardson, C. Touch-typing with a stylus. In Proc. INTERACT' '93 and CHI '93, 80-87.

11. Gutwin, C. Workspace awareness in real-time groupware environments. PhD. Thesis, University of Calgary, 1997.

12. Jansen, E. Teaching users how to interact with gesture-based interfaces; a comparison of teaching-methods. MSc thesis, Eindhoven University of Technology, 2012.

13. Kin, K., Hartmann, B., DeRose, T. Agrawala, M. Proton: multitouch gestures as regular expressions. In Proc. CHI '12, 2885-2894.

14. Kray, C., Nesbitt, D., Dawson, J., Rohs, M. User-defined gestures for connecting mobile phones, public displays, and tabletops. In Proc. MobileHCI '10, 239-248.

15. Kristensson, P.O., Zhai, S. Command strokes with and without preview: using pen gestures on keyboard for command selection. In Proc. CHI’07, 1137-1146.
16. Kurdyukova, E., Redlin, M., André, E. Studying user-defined iPad gestures for interaction in multi-display environment. In Proc. IUI '12, 93-96.

17. Kühnel, C., Westermann, T., Hemmert, F., Kratz, S., Müller, A., Möller, S. I'm home: Defining and evaluating a gesture set for smart-home control. IJHCS, V. 69, 11, Oct 2011, 693-704.

18. Leap Motion. http://leapmotion.com. Last.Acc. 2012-09-18.

19. Long, Jr., A.C., Landay, J.A., Rowe, L.A. Implications for a gesture design tool. In Proc CHI'99, 40-47.

20. Long, Jr., A.C., Landay, J.A., Rowe, L.A., Michiels, J. Visual similarity of pen gestures. In Proc. CHI '00, 360-367.

21. Lü, H., Li, Y. Gesture coder: a tool for programming multitouch gestures by demonstration. In Proc. CHI '12, 2875-2884

22. MacKenzie, I.S., Zhang, S.X. The immediate usability of graffiti. In Proc. GI '97, CIPS, 129-137.

23. Microsoft Corp., Using Gestures. http://bit.ly/OXOvT4. Last. Acc. 2012-09-19.

24. Morris, M.R., Huang, A., Paepcke A., Winograd, T. Cooperative gestures: multi-user gestural interactions for co-located groupware. In Proc. CHI '06, 1201-1210.

25. Morris, M.R. Wobbrock, J.O., Wilson, A.D. Understanding users' preferences for surface gestures. In Proc. GI'10, 261268.

26. Ni, T., Bowman, D., North, C. AirStroke: bringing unistroke text entry to freehand gesture interfaces. In Proc. CHI '11, 2473-2476.

27. North, C., Dwyer, T., Lee, B., Fisher, D., Isenberg, P. Robertson, G.,Inkpen, K. Understanding Multi-touch Manipulation for Surface Computing. In Proc. INTERACT '09, 236-249.

28. Rubine, D. 1991. Specifying gestures by example. In Proc. SIGGRAPH' '91, 329-337.

29. Oh, U. and Findlater, L. The challenges and potential of enduser gesture customization. In Proc. CHI '13. Forthcoming.

30. Ruiz, J., Li, Y. and Lank, E. User-defined motion gestures for mobile interaction. In Proc CHI'11, 197-206.

31. Schmidt, S., Nacenta, M.A., Dachselt, R., Carpendale, S., A set of multi-touch graph interaction techniques. In Proc. ITS'10, 113-116.

32. Sodhi, R., Benko, H. Wilson, A. LightGuide: projected visualizations for hand movement guidance. In Proc. CHI '12, 179188.

33. Vatavu, R.D. User-defined gestures for free-hand TV control. In Proc. EuroiTV'12, 45-48.

34. Wobbrock, J.O., Aung, H.H., Rothrock, B., Myers, B.A. Maximizing the guessability of symbolic input. In $\mathrm{CHI}$ '05 EA, 1869-1872.

35. Wobbrock, J.O., Morris, M.R. Wilson, A.D. User-defined gestures for surface computing. In Proc. CHI '09, 1083-1092.

36. Wobbrock, J.O., Wilson, A.D., Li, Y. Gestures without libraries, toolkits or training: a $\$ 1$ recognizer for user interface prototypes. In Proc. UIST '07, 159-168.

37. Wu, M., Shen, C., Ryall, K., Forlines, C., Balakrishnan, R. Gesture registration, relaxation, and reuse for multi-point direct-touch surfaces. In Proc. Tabletop'09.

38. Zhai, S. Kristensson, P.O. Shorthand writing on stylus keyboard. In Proc. CHI'03, 97-104.

39. Zhai, S., Kristensson, P.O., Appert, C., Andersen, T.H., Cao, X. 2012. Foundational issues in touch-surface stroke gesture design - an integrative review. Foundations and Trends in Human-Computer Interaction 5(2): 97-205. 